\title{
Ablative Radioiodtherapie des Schilddrüsenkarzinoms
}

\section{Standardisierte versus personalisierte Deeskalation der I-131 Aktivität}

\author{
M. Dietlein; A. Drzezga \\ Klinik und Poliklinik für Nuklearmedizin, Uniklinik Köln
}

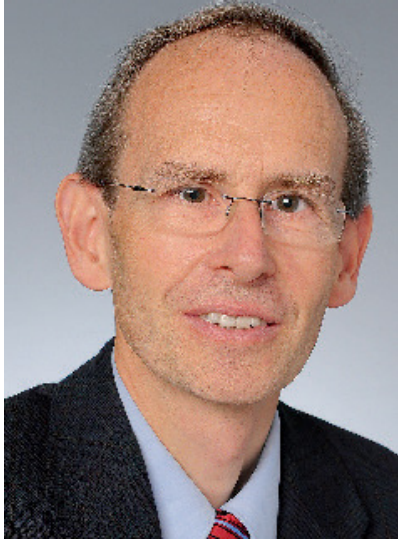

Prof. Dr. Markus Dietlein, Köln

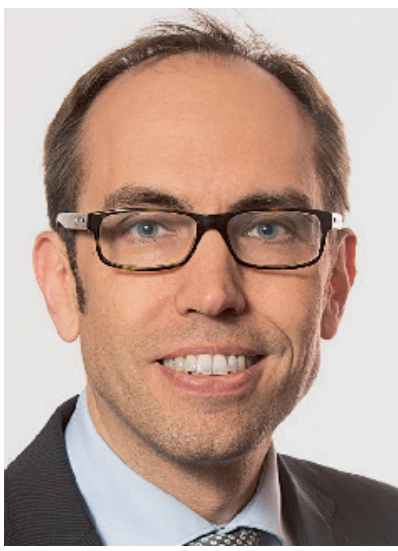

Prof. Dr. Alexander Drzezga, Köln
Der Nutzen einer ablativen Radioiodtherapie bei initial niedrigem oder intermediärem Rezidivrisiko eines differenzierten Schilddrüsenkarzinoms (DTC) mit dem Endpunkt eines verbesserten Gesamtüberlebens konnte aus den retrospektiven Analysen der National Cancer Database (USA) und des Surveillance, Epidemiology, and End Results (SEER)-Tumorregisters (USA) abgeleitet werden $(1,26,32)$. Andererseits wird anhand der SEER-Daten ein erhöhtes Leukämierisiko nach der Radioiodtherapie des DTC diskutiert (24), wenngleich Risikoangaben in absoluten Zahlen fehlen sowie die Kodierung der applizierten I-131 Aktivität und damit die wichtige Differenzierung zwischen der Ablation von Restschilddrüsengewebe und der hochdosierten Radioiodtherapie von Metastasen in dieser Arbeit nicht erfolgt ist (13, 43). Diese Differenzierung wäre jedoch für die Risikoabschätzung von entscheidender Bedeutung, da es zu kurz greift, ein dosisabhängiges Risiko pauschal auf die Radioiodtherapie insgesamt zu übertragen.

Die Existenz einer solchen Risikoschwelle, d.h. einer Abhängigkeit des Leukämierisikos von der Höhe der applizierten Aktivität, legen die Daten einer großen koreanischen Studie an 211360 Patienten nahe. Auf landesweiter Datenbasis wurde un-

Korrespondenzadresse

Prof. Dr. Markus Dietlein

Klinik und Poliklinik für Nuklearmedizin

Universitätsklinikum Köln

Kerpener Str. 62, 50937 Köln

Tel.: 02214785024

Nuklearmedizin 2018; 57: 118-123

https://doi.org/10.3413/Nukmed-0976-18-05

received: May 21, 2018

accepted: June 11, 2018 tersucht, oberhalb welcher I-131 Aktivität das Leukämierisiko einsetzt (36): Etwa die Hälfte der Patienten war mit I-131 behandelt worden. Es fand sich keine Risikoerhöhung bei kumulierten Aktivitäten bis 3,7 GBq I-131, wohl aber bei kumulierten Aktivitäten $\geq 5,5 \mathrm{GBq}$ I-131. Der Anteil der von einer Leukämie betroffenen Patienten in der Gruppe mit höher dosierter Radioiodtherapie war mit $0,06-0,07 \%$ immer noch sehr niedrig gegenüber den niedrig dosierten oder nicht mit Radiojod therapierten Patienten (Leukämierate 0,02\%). Das heißt 1 zusätzlicher Leukämiepatient auf 2000 Radioiodtherapien $>3,7 \mathrm{GBq}$ I-131 im Vergleich zu Patienten mit niedriger dosierter Radioiodtherapie bzw. im Vergleich zu Patienten ohne Radioiodtherapie (36).

Andere Daten aus Taiwan legen einen Risikoanstieg ebenfalls oberhalb von 5,5 GBq I-131 nahe (39). Eine kleinere monozentrische Studie an 2031 Patienten berechnete ein erhöhtes Risiko für Zweitmalignome oberhalb von kumuliert 7,4GBq I-131 (38). Das Risiko eines Sekundärmalignoms betrifft die Speicheldrüsen, den Magen, die Nieren und andere Organe (8-10). Ausgehend von der bei 416 Patienten gemessenen Organdosis an Speicheldrüsen, Magen, Nieren und Knochenmark errechnete sich strahlenbiologisch über die Lebensspanne eines Menschen extrapoliert für den Magen das höchste absolute Malignomrisiko (7). In dieser Modellrechnung verliefen die Risikokurven für die Aktivität $1,85 \mathrm{GBq}$ I-131 günstiger als die Kurven für die Aktivität 3,7 GBq I-131.

Der vorhergesagte Unterschied war für Patienten unter 30 Jahren besonders groß. Da die Strahlenexposition des Magens im Wesentlichen aus der Sekretion der Be- 
cherzeller von I-131 aus dem Bloodpool resultiert, wird die Applikationsform oral versus intravenös keine geeignete Strategie zur Strahlenminimierung sein. So bleibt die Frage, bei welchen Patienten mit $1,85 \mathrm{GBq} / 2 \mathrm{GBq} \mathrm{I}-131$ anstatt $3,7 \mathrm{GBq}$ I-131 ein exzellentes Therapieansprechen nach den Kriterien der American Thyroid Association (ATA) erzielt werden kann, ohne die Rate an zweizeitigen Ablationen zu erhöhen $(6,11,19,22)$. Die ATA hat das exzellente Therapieansprechen über ein stimuliertes Thyreoglobulin $(\mathrm{Tg}) \leq 1,0 \mathrm{ng} / \mathrm{ml}$ bzw. über ein nicht messbares Thyreoglobulin unter der TSH-Suppression definiert, während Patienten mit einer, ,indeterminate Response" Thyreoglobulin-Spannbreiten unter TSH-Stimulation über $1,0-10,0 \mathrm{ng} / \mathrm{ml}$ bzw. unter TSH-Suppression im unteren Assay-spezifischen Messbereich, aber $\leq 1,0 \mathrm{ng} / \mathrm{ml}$ aufweisen (15). Sofern noch höhere Thyreoglobulin-Spiegel messbar sind, sprechen die ATA-Guidelines von einer inkompletten biochemischen Remission, sofern bildmorphologisch bzw. klinisch kein Tumorgewebe zu lokalisieren ist.

\section{Standardisierung bei der I-131 Aktivitätsreduktion}

Die beiden randomisierten Studien ESTIMABL (35) und HiLo (22) spielen in den Leitlinien der ATA und der British Thyroid Association (BTA) eine zentrale Rolle für die Empfehlung von 1,1 GBq I-131 gegenüber 3,7 GBq I-131 bei der ablativen Radioiodtherapie $(15,28)$. Da in beiden Studien der primäre Endpunkt einer erfolgreichen Ablation durch ein Tg unter rekombinantem humanem TSH $(\mathrm{rhTSH}) \leq 2,0 \mathrm{ng} / \mathrm{ml}$ definiert war, umfasst der Ablationserfolg auch solche Patienten, die nach aktuellen ATA-Kriterien der „indeterminate Respon$s e^{\text {" }}$ zugerechnet werden. In der HiLo-Studie wurde in den beiden Studienarmen mit 1,1 GBq I-131 zudem über eine erhöhte Rate an zweiten ablativen Radioiodtherapien ( $9 \%$ versus $4 \%$ bei Applikation von 3,7 GBq I-131) berichtet. Erhöhte Raten an zweiten ablativen Radioiodtherapien in den Behandlungsarmen mit 1,1 GBq I-131 fanden sich in 3 weiteren randomisierten Studien, die differente Ablationsaktivitäten verglichen $(6,11,19)$. In einer weiteren
Studie mit einem Vergleich von $1,1 \mathrm{GBq}$ versus 5,5GBq I-131 war nicht die Therapieaktivität, sondern der Thyreoglobulin (Tg)-Spiegel vor der Ablation entscheidend für das spätere Tg-basierte Therapieansprechen (3). Die Nicht-Unterlegenheit von $1,1 \mathrm{GBq}$ versus $3,0 \mathrm{GBq}$ I-131 zeigte eine kleinere koreanische Studie mit Fokus auf Tumorstadien mit einer mikroskopischen extrathyreoidalen Infiltration und mit einer nodalen Metastasierung in das zervikozentrale Kompartiment (pT1a) (37). In der Mehrzahl der Studien aber führte das 1,1 GBq-Konzept letztlich zu einer verlängerten mittleren Behandlungsdauer aufgrund einer erhöhten Rate an Zweitablationen.

Ein naheliegender Zwischenweg zu diesen beiden Dosiskonzepten hat sich in Deutschland zum Teil bereits in der Patientenversorgung etabliert: Ausgehend von vergleichenden Daten aus der Universitätsklinik LMU München (42) und aus dem Universitätsklinikum Rostock (16) lässt sich ein exzellentes Therapieansprechen mit $2 \mathrm{GBq}$ I-131 ebenso häufig erreichen wie mit 3,7 GBq I-131. Dabei war in beiden Studien die Messlatte zum Ablationserfolg mit einem stimulierten $\mathrm{Tg} \leq 0,5 \mathrm{ng} / \mathrm{ml}$ etwa 6 Monate nach der ablativen Radioiodtherapie sogar höher angelegt als in den ATAKriterien für ein exzellentes Therapieansprechen. Die Kohortenstudien dokumentieren eine erfolgreich umgesetzte Deeskalationsstrategie standardisiert für ein Mischkollektiv aus low-risk und intermediate-risk Patienten, wobei in dem Kollektiv der LMU München keine Patienten mit nachgewiesener Lymphknotenmetastasierung enthalten waren $(16,42)$.

\section{Perspektiven zur Perso- nalisierung der I-131 Akti- vitätsreduktion}

Bereits in den ATA-Guidelines beschrieben Haugen und Mitarb. (15) einen Korridor für eine personalisierte Therapieberatung, indem ein stimuliertes $\mathrm{Tg} \leq 1,0 \mathrm{ng} / \mathrm{ml}$ vor der ablativen Radioiodtherapie als prognostisch sehr günstig zu werten ist bzw. ein postoperativ stimuliertes $\mathrm{Tg}>5-6 \mathrm{ng} / \mathrm{ml}$ das Risiko für einen ausbleibenden Ablationserfolg um den Faktor 5 erhöht, falls ein
Niedrigdosiskonzept mit 1,1 GBq I-131 angewandt wird. Postoperativ stimulierte TgSpiegel $>5,6 \mathrm{ng} / \mathrm{ml}$ gingen in einer aktuelleren Studien bereits mit einem 2,38-fach erhöhten Rezidivrisiko einher (25). Bei der Interpretation eines Tg-Spiegels vor der ablativen Radioiodtherapie ist zu beachten:

- Der Nadir des Tg wird erst 3-4 Wochen nach einer Thyreoidektomie erreicht.

- Nicht geeignet ist der Tg-Wert als initialer Prädiktor für etwa $20 \%$ der Patienten mit erhöhten Tg-Antikörpern, wobei dieser Prozentwert bei vergleichender Analyse der Tg-Antikörper Assays dreier Hersteller bestätigt wurde (12).

- Messbare Tg-Spiegel entstehen entweder durch Schilddrüsen-Reste oder durch Tumorpersistenz. Die Höhe des Tg-Spiegels wird zudem durch das Ausmaß der TSH-Stimulation beeinflusst.

Insofern ist ein stimulierter $\mathrm{Tg}$-Spiegel $\leq 1,0 \mathrm{ng} / \mathrm{ml}$ vor der I-131 Ablation ein Hinweis auf ein operationstechnisch gelungenes, hohes Resektionsausmaß in Kombination mit einer sehr niedrigen Wahrscheinlichkeit einer Metastasierung. Ein postoperativ niedriger Tg-Wert identifiziert in einem personalisierten Konzept die Patienten, die grundsätzlich für Aktivitäten um 2GBq I-131 geeignet sein dürften. Bei Anwendung von $2 \mathrm{GBq}$ I-131 zur Ablation (Deeskalation der I-131 Aktivität) darf zudem anhand der anatomischen und funktionellen Bildgebung (Sonographie, I-123 Szintigraphie, I-123 SPECT/CT, I-124 PET/ CT) nicht der Verdacht auf eine Lymphknotenmetastasierung bestehen.

Bei höherem Tg-Spiegel ist der Umkehrschluss schwieriger wegen der vielfältigen Einflussgrößen auf einen messbaren Tg-Wert, wie Tg-Halbwertszeit (1-3 Tage), der Zeitspanne bis zum Erreichen des TgNadirs sowie der Möglichkeit von Schilddrüsenresten und/ oder der Tumorpersistenz. Für die Interpretation höherer TgSpiegel ist wiederum die anatomische und funktionelle Bildgebung (Sonographie, Tc-99m Szintigraphie, Tc-99m SPECT/CT, I-123 Szintigraphie, I-123 SPECT/CT, I-124 PET/CT) wichtig, da sich die I-131 Aktivitäten zur ablativen Radioiodtherapie des Schilddrüsenrestgewebe in Abgrenzung zur Radioiodtherapie bei konkretem 
Metastasenverdacht (Eskalation der I-131 Aktivität) unterscheiden.

Die Studien, die den Zusammenhang zwischen dem Tg-Spiegel vor der ablativen Radioiodtherapie und dem Therapieansprechen nach 6-12 Monaten untersucht haben, unterscheiden sich sowohl im Ausgangspunkt (nicht-stimulierter versus stimulierter Tg-Wert vor der I-131 Ablation) als auch im Endpunkt (Erfolgsdefinition über die „exzellente Response“ versus einer Summation aus exzellenter plus ,indeterminate Response").

\section{2 a. Ausgangspunkt nicht- stimulierter Tg-Spiegel vor I-131 Ablation}

In einer prospektiven Studie mit 85 Patienten war der nicht-stimulierte Tg-Wert $\leq 1,8 \mathrm{ng} / \mathrm{ml}$ ein Prädiktor für ein Therapieansprechen, wobei zur Ablation 3,7 GBq I-131 eingesetzt wurden (30). Allerdings war der spätere Therapieerfolg über ein nicht-stimuliertes $\mathrm{Tg}<1 \mathrm{ng} / \mathrm{ml}$ definiert, was nach den ATA-Kriterien der Summe aus exzellenter und „indeterminate Respon-

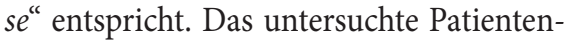
kollektiv war dem mittleren Rezidivrisiko mit zusätzlichen Risikofaktoren wie aggressiver Histologie, Lymphknotenmetastasen $>1,5 \mathrm{~cm}$, extranodaler Infiltration bzw. mit der Kombination aus mehreren Risikofaktoren zuzuordnen. Wenn eine personalisierte Strategie für Deutschland den Anspruch auf ein exzellentes Therapieansprechen vertritt, wurden in dieser brasilianischen Studie hierzu nicht kompatible Erfolgskriterien verwendet.

Die gleiche Autorengruppe präsentierte eine zweite prospektive Studie mit $102 \mathrm{~Pa}$ tienten bei gleichem Risikoprofil, aber veränderten Bedingungen im Ausgangspunkt und im Endpunkt (31). Ausgangspunkt war das nicht-stimulierte $\mathrm{Tg}<0,3 \mathrm{ng} / \mathrm{ml}$ vor der I-131 Ablation, Endpunkt ein nicht-stimuliertes Tg $\leq 0,2 \mathrm{ng} / \mathrm{ml}$, entsprechend einer „exzellenten Response“ nach ATA-Kriterien. Die Therapieaktivität lag bei 1,1 GBq oder 1,85 GBq I-131 zur ablativen Radioiodtherapie. Mit diesen I-131 Aktivitäten wurde ein exzellentes Therapieansprechen bei 99\% der Patienten erzielt. Die Autoren schlussfolgerten, dass ein niedriger, nicht-stimulierter Tg-Spiegel (hier $\mathrm{Tg}<0,3 \mathrm{ng} / \mathrm{ml}$ ), gemessen mit modernen Tg-Assays, ein geeignetes Kriterium für eine Aktivitätsreduktion bei Patienten mit intermediärem Rezidivrisiko darstellt.

\section{2 b. Ausgangspunkt stimu- lierter Tg-Spiegel vor I-131 Ablation}

In einer Metaanalyse von 15 Studien wurde die Höhe des Tg-Spiegels $\geq 4$ Wochen postoperativ als Prädiktor für ein späteres Therapieansprechen untersucht (45). Anhand der ROC-Kurve und dem Forest-Plot errechnete sich ein stimulierter Tg-Grenzwert von postoperativ $\leq 5,8 \mathrm{ng} / \mathrm{ml}$, um ein späteres Therapieansprechen auf die operative und nuklearmedizinische Therapie vorherzusagen. Die ablative Radioiodtherapie wurde mit Aktivitäten zwischen 1,1 und 5,5GBq I-131 durchgeführt. Das Therapieansprechen war über die Summe aus "exzellenter Response" und "indeterminate Response" gemäß ATA-Kriterien definiert. Der gleichen Frage widmete sich eine aktuelle Studie aus Japan (17): Hier war ein TgGrenzwert von $\leq 5,4 \mathrm{ng} / \mathrm{ml}$ postoperativ unter rhTSH geeignet, um nach einer Ablation mit 1,1 GBq I-131 eine „exzellente Response" oder eine "indeterminate Response“ $\mathrm{zu}$ erzielen, wobei in der japanischen Studie der Bereich der ,indeterminate Respon$s e$ " auf ein $\mathrm{Tg} / \mathrm{rhTSH} \leq 5 \mathrm{ng} / \mathrm{ml}$ eingegrenzt wurde. Aufgrund der Wertung von „exzellente Response“ und „indeterminate Respon-

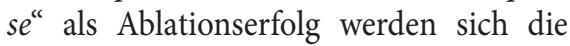
Schlussfolgerungen aus der Metaanalyse und aus der japanischen Studie nicht auf eine Handlungsempfehlung in Deutschland übertragen lassen. Wenn aus der Perspektive der Nuklearmedizin in Deutschland als Ablationserfolg ein exzellentes Therapieansprechen nach einzeitiger ablativer Radioiodtherapie gefordert wird, sind offenbar Tg-Spiegel vor der I-131 Ablation um 5-6ng/ml als Entscheidungsschwelle für die I-131 Aktivitätsbemessung ungeeignet.

Eine koreanische Studie beschränkte die Definition des Ablationserfolges auf die „exzellente Response" gemäß den ATA-Kriterien (27). In der ROC-Kurve war ein Tg/ rhTSH $\leq 2,0 \mathrm{ng} / \mathrm{ml} 4$ Wochen vor der I-131
Ablation am besten geeignet, um einen späteren Behandlungserfolg der ablativen Radioiodtherapie vorauszusagen. Eingesetzt wurden $2,96-6,66 \mathrm{GBq}$ I-131 unter rhTSH.

Eine chinesische Studie mit 398 Patienten verwendete ein stimuliertes $\mathrm{Tg}<2 \mathrm{ng} /$ $\mathrm{ml}$ vor der I-131 Ablation, um ein späteres exzellentes Therapieansprechen gemäß der ATA-Definition vorauszusagen (47). In der multivariaten Analyse erwies sich der stimulierte Tg-Spiegel vor der I-131 Ablation als einziger signifikanter Prädiktor für ein exzellentes Therapieansprechen. Damit war der stimulierte Tg-Spiegel vor der I-131 Ablation (personalisiertes Konzept) ein besserer Prädiktor als das initiale TNM-Stadium (standardisiertes Konzept). Eine exzellente Response nach der I-131 Ablation erzielten 92,2\% der Patienten, wobei die Erfolgsrate in der Subgruppe mit einem stimulierten $\mathrm{Tg}<1 \mathrm{ng} / \mathrm{ml}$ erwartungsgemäß noch höher lag als in der Gruppe, die über ein stimuliertes $\mathrm{Tg}$ von $1 \mathrm{ng} / \mathrm{ml}$ bis $<2 \mathrm{ng} / \mathrm{ml}$ definiert war (Odds ratio 2,659). Die ablative Radioiodtherapie wurde risikoadaptiert mit $1,85-5,5 \mathrm{GBq}$ I-131 in Hypothyreose durchgeführt. Insofern enthält diese Studie keinen systematischen Vergleich verschiedener I-131 Aktivitäten.

In einer retrospektiven Auswertung von Bandeira et al. (5) hatten die Patienten mit exzellentem Therapieansprechen, gemessen 1 Jahr nach der ablativen Radioiodtherapie, vor der Ablation ein stimuliertes $\mathrm{Tg}$ von $2,1 \pm 3,3 \mathrm{ng} / \mathrm{ml}$ gezeigt. Erwartungsgemäß höhere stimulierte Tg-Spiegel von $8,2 \pm 9,2 \mathrm{ng} / \mathrm{ml}$ fanden sich vor der I-131 Ablation bei Patienten, die nach 1 Jahr der „indeterminate Response“ zugeordnet wurden.

\section{Zeitspanne bis zur I-131 Ablation}

Wird nach der Thyreoidektomie mit der ablativen Radioiodtherapie unter Verordnung von Levothyroxin und rhTSH gewartet - auch um ggf. den Tg-Spiegel als Entscheidungsfaktor für die I-131 Aktivitätsbemessung nutzen zu können - existieren inzwischen Daten, inwieweit die Dauer des Aufschubs bis zur I-131 Ablation mit dem 
Therapieansprechen korreliert sind. Nach einer Studie aus China (20) stieg mit einem Verzug $>3$ Monaten der Patientenanteil mit einer ,indeterminate Response" an. Die Ursache für diese Beobachtung bleibt unklar.

Konsistent zu der früheren dosimetrischen Arbeit aus Würzburg (14) bestätigten sich unter rhTSH eine Verlängerung der effektiven HWZ von I-131 im Vergleich zur Hormonkarenz in einer weiteren Studie aus China (18). Dieser Effekt betraf sowohl Schilddrüsenrestgewebe als auch Lymphknotenmetastasen: Die effektive HWZ im Schilddrüsenrestgewebe lag in der Hypothyreose bei 19h (Spannbreite der Interquartile $16,8-23,1 \mathrm{~h}$ ) und unter rhTSH bei $33 \mathrm{~h}(23,7-43,3 \mathrm{~h})$ bzw. in Lymphknotenmetastasen in der Hypothyreose bei $11,8 \mathrm{~h}(11,0-13,3 \mathrm{~h})$ und unter rhTSH bei $23,9 \mathrm{~h}(16,8-27,7 \mathrm{~h})$. Dieser unerwartet signifikante Effekt zugunsten von rhTSH war in der Studie von Hänscheid und Mitarb. im Schilddrüsenrestgewebe in der Hypothyreose mit 48,0h $\pm 52,6 \mathrm{~h}$ (Standardabweichung) und unter rhTSH mit $67,6 \mathrm{~h} \pm 48,8 \mathrm{~h}$ quantifiziert worden (14).

\section{Konsequenzen diverser Neuklassifikationen für die I-131 Ablation}

Mit der 8. Auflage der TNM-Klassifikation des American Joint Committee on Cancer (AJCC) führt eine minimale Tumorinfiltration in das perithyreoidale Fettgewebe nicht mehr zu einer Höherstufung des pTStadiums (44). Basierend auf den Daten der National Cancer Database (USA) und des SEER-Registers (USA) bewirkt der mikroskopische Kapseldurchbruch keine Risikoerhöhung (4) bzw. stellt mit einer $\mathrm{Ha}$ zard ratio von 1,13 einen nur moderaten Risikofaktor für ein geringeres Gesamtüberleben dar (46). Dies deckt sich mit den früheren Daten aus der multizentrischen MSDS-Studie in Deutschland (29). In der Konsequenz sollte der nur mikroskopisch nachgewiesene Durchbruch der Schilddrüsenkapsel nicht zu erhöhten I-131 Aktivitäten bei der ablativen Radioiodtherapie des differenzierten Schilddrüsenkarzinoms führen.
Aus der WHO-Klassifikation 2017 ging eine neue Gruppe gekapselter follikulärer Tumoren mit unklarem malignem Potenzial hervor, wie z.B. das „Noninvasive follicular thyroid neoplasm with papillary-like nuclear feature" (NIFTP) (21). Nachdem im Verlauf über mehr als 10 Jahren keiner aus einer Kohorte von 94 Patienten ein Lokalrezidiv oder eine Metastasierung erlitt (40), sollte diese Tumorentität nicht der ablativen Radioiodtherapie zugeführt werden. Allerdings wurde in der Abgrenzung eines NIFTP versus der follikulären Variante eines PTC (eFVPTC) bei einem „Ringversuch" mit weltweit 4 Instituten für Pathologie im Grad der Übereinstimmung ein Kappa-Wert um 0,6 erzielt, womit die Reproduzierbarkeit der Bewertung als mäßig einzustufen ist (41). Im Zweifel kann eine Referenzpathologie hilfreich sein; der Patient erhält währenddessen eine Levothyroxin-Substitution.

Die initiale Risikoklassifikation (low intermediate - high) versteht die ATA als ein Kontinuum. Wie sind Patienten mit Lymphknotenmetastasen einzuordnen? Die prognostisch günstigste Subgruppe sind Patienten mit Mikrometastasen (definitionsgemäß bis $0,2 \mathrm{~cm}$ ) in bis $\mathrm{zu} 5$ Lymphknoten mit einem Risiko von 5\% für ein späteres strukturelles Rezidiv. Diese Risikoangabe der ATA ist vergleichbar mit dem Rezidivrisiko eines Patienten mit minimaler extrathyreoidaler Infiltration (Risikoangabe der ATA 3-8\%). Diese beiden pathologischen Befunde ordnete die ATA der low-risk Gruppe zu (15).

Beide initialen pathologischen Befunde (wenige Mikrometastasen, minimale extrathyreoidale Infiltration) eignen sich grundsätzlich für ein 2GBq I-131 Ablationskonzept, wenn postoperativ günstige Voraussetzungen anhand des Tg-Spiegels, der Sonographie und szintigraphischer Verfahren nachgewiesen sind.

Die Analyse der National Cancer Database und des SEER-Registers zeigen, dass das Rezidivrisiko mit der Anzahl der Lymphknotenmetastasen eher kontinuierlich ansteigt (2) und dass bei der Risikostratifikation nodal-positiver Patienten kein dezidierter Grenzwert für die Anzahl an Lymphknotenmetastasen existiert. Bei Nachweis von Lymphknotenmetastasen sind die Patienten mit zahlreichen Lymph- knotenmetastasen, mit extranodaler Kapselüberschreitung oder mit Befall des lateralen Halskompartiments (pN1b) besonders gefährdet.

In einer aktuellen monozentrischen Studie an 1653 Patienten mit papillärem Schilddrüsenkarzinom (mittleres Erkrankungsalter mit 68 Jahren relativ hoch) lag die Tumor-assoziierte Mortalität bei 0,2\% für die Patienten mit einem N0-Status und bei $0,3 \%$ für $\mathrm{pNla}$, aber bei 3,0\% für den pN1b-Status $(33,34)$. Beträgt die Relation aus befallenen Lymphknoten zu den insgesamt operativ entfernten Lymphknoten $\geq 0,31$, stieg in einer chinesischen Studie die Hazard ratio für ein Rezidiv auf $>11$ (48).

All diese Erkenntnisse zeigen, dass die systematische Lymphadenektomie für die Validität einer personalisierten Deeskalation bzw. Eskalation der I-131 Aktivität günstig ist, während die Entfernung nur einzelner vergrößerter Lymphknoten eher auf eine standardisierte Anwendung der I-131 Therapieaktivität hinausläuft. Andererseits ist bei den niedrigen lokalen $\mathrm{Tu}$ morstadien pT1-2 auch nach Verzicht auf eine Lymphadenektomie bei einem Lymphknotenstatus cN0 eine Personalisierung der I-131 Ablationsaktivität anhand des Tg-Spiegels, der Sonographie und der szintigraphischen Verfahren sinnvoll.

\section{Perspektiven für eine Deeskalationsstrategie}

Dem diskutierten Risiko für Sekundärneoplasien bei Verwendung von $\geq 5,5 \mathrm{GBq}$ I-131 kann die Nuklearmedizin gut begründete Deeskalationsstrategien bei der ablativen Radioiodtherapie entgegensetzen. Die ablative Radioiodtherapie mit 2 GBq I-131 bietet in einem standardisierten Konzept bei Patienten mit niedrigem Rezidivrisiko, wahrscheinlich auch bei intermediärem Rezidivrisiko, gute Aussichten auf ein späteres exzellentes Therapieansprechen. Nachdem mit einer Aktivität von 3,7 GBq I-131 keine besseren Ablationserfolge als mit $2 \mathrm{GBq}$ I-131 dokumentiert wurden $(16,42)$, bietet sich - unter dem Aspekt der Risikominimierung - das $2 \mathrm{GBq}-$ Konzept an $(8,9)$. 
Eine personalisierte Strategie bei der Aktivitätsreduktion ist insbesondere für die Patienten mit intermediärem Risikoprofil zu erwägen. Einem intermediärem Risikoprofil werden in den ATA-Guidelines die Patienten im initialen Stadium pT3 und/ oder pN1 (außer nodale Mikrometastasen als "low-risk" Befund) zugeordnet (15). Falls der postoperativ gemessene Tg-Spiegel bei TSH-Stimulation unter $2 \mathrm{ng} / \mathrm{ml}$ bzw. ohne eine TSH-Stimulation unter $0,3 \mathrm{ng} / \mathrm{ml}$ bleibt, wird für die so ausgewählten Patienten durch einzeitig 2 GBq I-131 mit hoher Wahrscheinlichkeit ein exzellentes Therapieansprechen zu erzielen sein. Die Voraussetzung, dass möglichst viele Patienten personalisiert durch eine ablative Radioiodtherapie mit $2 \mathrm{GBq}$ I-131 erfolgreich behandelt werden können, schafft der Operateur mit hoher Expertise. Die Überschrift eines Review fasst es kurz zusammen: No longer ,one size fits all“ (23).

Fazit: Wenn postoperativ anhand des Tg-Spiegels, der Sonographie und szintigraphischer Verfahren günstige Voraussetzungen nachgewiesen sind, dürfte sich das für „low-risk“ Patienten bereits etablierte 2 GBq I-131 Ablationskonzept auch für Patienten mit intermediärem Risikoprofil eignen.

\section{Literatur}

1. Adam MA, Pura J, Gu L et al. Extent of surgery for papillary thyroid cancer is not associated with survival. An analysis of 61,775 patients. Ann Surg 2014; 260: 601-607.

2. Adam MA, Pura J, Goffredo P et al. Presence and number of lymph node metastases are associated with compromised survival for patients younger than age 45 years with papillary thyroid cancer. J Clin Oncol 2015; 33: 2370-2375.

3. Aghaei A, Ayati N, Shafiei S et al. Comparison of treatment efficacy 1 and 2 years after thyroid remnant ablation with 1110 versus $5550 \mathrm{MBq}$ of iodine-131 in patients with intermediate risk differentiated thyroid cancer. Nucl Med Commun 2017; 38: 927-931.

4. Al-Qurayshi Z, Shama MA, Randolph GW, Kandil E. Minimal extrathyroidal extension does not affect survival of well-differentiated thyroid cancer. Endocr Relat Cancer 2017; 24: 221-226.

5. Bandeira L, Padovani RDP, Ticly AL et al. Thyroglobulin levels before radioactive iodine therapy and dynamic risk stratification after 1 year in patients with differentiated thyroid cancer. Arch Endocrinol Metab 2017; 61: 590-599.

6. Caglar M, Bozkurt FM, Akca CK et al. Comparison of 800 and $3700 \mathrm{MBq}$ iodine-131 for the postoperative ablation of thyroid remnant in patients with low-risk differentiated thyroid cancer. Nucl Med Commun 2012; 33: 268-274.

7. Corréa NL, Vasconcellos de Sa L, Ramalho de Mello RC. Estimation of second primary cancer risk after treatment with radioactive iodine for differentiated thyroid carcinoma. Thyroid 2017; 27: 261-270.

8. Dietlein M, Drzezga A. Ablative Radioiodtherapie bei niedrigem und intermediärem Rezidivrisiko. Höhere Überlebensrate beim Schilddrüsenkarzinom. Nuklearmedizin 2016; 55: 71-76.

9. Dietlein M, Eschner W, Grünwald F, Lassmann M, Verburg FA, Luster M. Procedure guidelines for radioiodine therapy of differentiated thyroid cancer (version 4). [German]. Nuklearmedizin 2016; 55: 77-89.

10. Dietlein M, Drzezga A. Geschmacksstörungen (Dysgeusie) und Radioiodtherapie des Schilddrüsenkarzinoms. Vorschädigung durch Antidepressiva und Sedativa beachten. Nuklearmedizin 2017; 56: 125-131.

11. Fallahi B, Beiki D, Takavar A, Fard-Esfahani A, Gilani KA, Saghari M, Eftekhari M. Low versus high radioiodine dose in postoperative ablation of residual thyroid tissue in patients with differentiated thyroid carcinoma: a large randomized clinical trial. Nucl Med Commun 2012; 33: 275-282.

12. Giovanella L, Imperiali M, Verburg FA, Trimboli P. Early post-treatment risk stratification of differentiated thyroid cancer: comparison of three highsensitive Tg assays. Eur J Endocrinol 2018; 187: 77-84.

13. Greenspan BS. Radioiodine treatment of well-differentiated thyroid cancer: balancing risks and benefits. J Clin Oncol 2018; doi: 10.1200/JCO.2018.78.6384

14. Haenscheid H, Lassmann M, Luster M et al. Iodine biokinetics and dosimetry in radioiodine therapy of thyroid cancer: procedures and results of a prospective international controlled study of ablation after rhTSH or hormone withdrawal. J Nucl Med 2006; 47 : 648-654.

15. Haugen BR, Alexander EK, Bible KC et al. 2015 American Thyroid Association management guidelines for adult patients with thyroid nodules and differentiated thyroid cancer. Thyroid 2016; 26: 1-133.

16. Heuschkel M, Fruechte K, Becker J et al. Effektivität verschiedener Iod-131-Aktivitäten zur Restgewebsablation beim differenzierten Schilddrüsenkarzinom [Abstract]. Nuklearmedizin 2018; 57: A28.

17. Higushi T, Achmad A, Binh DD et al. Determining patient selection tool and response predictor for outpatient $30 \mathrm{mCi}$ radioiodine ablation dose in non-metastatic differentiated thyroid carcinoma: a Japanese perspective. Endocrine J 2018; doi:10.1507/endocrij.EJ17-0343.

18. Hong CM, Kim C-Y, Son SH et al. I-131 biokinetics of remnant normal thyroid tissue and residual thyroid cancer in patients with differentiated thyroid cancer: comparison between recombinant human TSH administration and thyroid hormone withdrawal. Ann Nucl Med 2017: 31: 582-589.

19. Kukulska A, Krajewska J, Gawkowska-Suwinska M et al. Radioiodine thyroid remnant ablation in patients with differentiated thyroid carcinoma (DTC): prospective comparison of long-term out- comes of treatment with 30,60 and $100 \mathrm{mCi}$. Thyroid Research 2010; 3: 9 .

20. Li H, Zhang Y, Wang C et al. Delayed initial radioiodine therapy related to incomplete response in low- to intermediate-risk differentiated thyroid cancer. Clin Endocrinol, published online 2018, January 16; doi: 10.1111/cen.13551.

21. Lloyd RV, Osamura RY, Klöppel G, Rosai J. WHO Classification of Tumours of Endocrine Organs. Fourth Edition. Volume 10. International Agency for Research on Cancer (IARC), World Health Organization (WHO). 2017; ISBN-13 (print book): 978-92-832-4493-6. www.iarc.fr

22. Mallick U, Harmer C, Yap B et al. Ablation with low-dose radioiodine and thyrotropin alfa in thyroid cancer. N Engl J Med 2012; 366: 1674-1685.

23. Marti JL, Morris LGT, Ho AS. Selective use of radioactive iodine (RAI) in thyroid cancer: no longer "one size fits all". Eur J Surg Oncol 2018; 44: 348-356.

24. Molenaar RJ, Sidana S, Radivoyevitch T et al. Risk of hematologic malignancies after radioiodine treatment of well-differentiated thyroid cancer. J Clin Oncol, published online December 18, 2017; doi.org/10.1200/JCO.2017.75.0232.

25. Mousa U, Yilmaz SA, Nar A. Stimulated thyroglobulin values above $5.6 \mathrm{ng} / \mathrm{ml}$ before radioactive iodine ablation treatment following levothyroxine withdrawal is associated with a 2.38 -fold risk of relapse in $\mathrm{Tg}$-ab negative subjects with differentiated thyroid cancer. Clin Trans Oncol 2017; doi: 10.1007/s12094-017-1640-3.

26. Orosco RK, Hussain T, Brumund KT et al. Analysis of age and disease status as predictors of thyroid cancer-specific mortality using the surveillance, epidemiology, and end results database. Thyroid 2015; 25: 125-132.

27. Park HJ, Min J-J, Bom H-S et al. Early stimulated thyroglobulin for response prediction after recombinant human thyrotropin-aided radioiodine therapy. Ann Nucl Med2017; 31: 616-622.

28. Perros P, Colley S, Boelart K et al. British Thyroid Association guidelines for the management of thyroid cancer. Clin Endocrinol (Oxf) 2014; 81 (Suppl. 1): 1-122.

29. Riemann B, Krämer JA, Schmid KW, Dralle H, Dietlein M, Schicha H, Sauerland C, Frankewitsch T, Schober O. Risk stratification of patients with locally aggressive differentiated thyroid cancer: results of the MSDS trial. Nuklearmedizin 2010; 49: 79-84.

30. Rosario PW, Mourao GF, Calsolari MR. Efficacy of adjuvant therapy with $3.7 \mathrm{GBq}$ radioactive iodine in intermediate-risk patients with higher risk features and predictive value of postoperative nonstimulated thyroglobulin. Nucl Med Commun 2016; 37: 1148-1153.

31. Rosario PW, Mourao GF, Calsolari MR. Low postoperative nonstimulated thyroglobulin as a criterion for the indication of low radioiodine activity in patients with papillary thyroid cancer of intermediate risk with higher risk features. Clin Endocrinol 2016; 85: 453-458.

32. Ruel E, Thomas S, Dinan M et al. Adjuvant radioactive iodine therapy is associated with improved survival for patients with intermediate-risk papillary thyroid cancer. J Clin Endocrinol Metab 2015; 100: 1529-1536. 
33. Sapuppo G, Palermo F, Russo M et al. Latero-cervical lymph node metastases (N1b) represent an additional risk factor for papillary thyroid cancer outcome. J Endocrinol Invest 2017; 40: 1355-1363.

34. Sapuppo G, Tavarelli M, Russo M et al. Lymph node location is a risk factor for papillary thyroid cancer-related death. J Endocrinol Invest 2018; doi: 10.1007/s40618-018-0865-5.

35. Schlumberger M, Catargi B, Borget I et al. Strategies of radioiodine ablation in patients with low risk thyroid cancer. N Engl J Med 2012; 366: 1663-1673.

36. Seo GH, Cho YY, Chung JH, Kim SW. Increased risk of leukemia after radioactive iodine therapy in patients with thyroid cancer: a nationwide, population-based study in Korea. Thyroid 2015; 25 927-934.

37. Seo M, Kim YS, Lee JC et al. Lowe-dose radioactive iodine ablation is sufficient in patients with small papillary thyroid cancer having minor extrathyroidal extension and central lymph node metastasis (T3 N1a). Clin Nucl Med 2017; 42: 842-846.

38. Silva-Vieira M, Carrilho Vaz S, Esteves S et al. Second primary cancer in patients with differentiated thyroid cancer: does radioiodine play a role? Thyroid 2017; 27: 1068-1076.

39. Teng CJ, Hu YW, Chen SC. Use of radioactive iodine for thyroid cancer and risk of second primary malignancy: a nationwide population-based study. J Natl Cancer Inst 2015 Nov 3; 108(2). pii: djv314. doi: 10.1093/jnci/djv314.

40. Thompson LDR. Ninety-four cases of encapsulated follicular variant of papillary thyroid carcinoma: a name change to Noninvasive Follicular Thyroid Neoplasm with Papillary-like Nuclear Features would prevent overtreatment. Modern Pathol 2016; 698-707.

41. Thompson LDR, Poller DN, Kakudo K et al. An international interobserver variability reporting of the nuclear scoring criteria to diagnose Noninvasive Follicular Thyroid Neoplasm with Papillarylike Nuclear Features: a validation study. Endocrine Pathol 2018; doi org/10.1007/s12022-018-9520-0.

42. Todica A, Haidvogl S, Fendler WP et al. Effectiveness of reduced radioiodine activity for thyroid remnant ablation after total thyroidectomy in patients with low to intermediate risk differentiated thyroid carcinoma. Nuklearmedizin 2017; 56 211-218.

43. Tulchinsky M, Binse I, Campenni A et al. Radioactive iodine therapy for differentiated thyroid cancer: lessons from confronting controversial literature on risks for secondary malignancy. J Nucl Med 2018; doi: 10.2967/jnumed.118.211359.
44. Tuttle RM, Haugen B, Perrier ND. Updated American Joint Committee on Cancer/ Tumor Node - Metastasis staging system for differentiated and anaplastic thyroid cancer (eighth edition): what changed and why? Thyroid 2017; 751-756.

45. Webb RC, Howard RS, Stojadinovic A et al. The utility of serum thyroglobulin measurement at the time of remnant ablation for predicting diseasefree status in patients with differentiated thyroid cancer: a meta-analysis involving 3947 patients. J Clin Endocrinol Metab 2012; 97: 2754-2763.

46. Youngwirth LM, Adam MA, Scheri RP et al. Extrathyroidal extension is associated with compromised survival in patients with thyroid cancer. Thyroid 2017; 27: 626-631.

47. Zhang Y, Hua W, Zhang X et al. The predictive value for excellent response to initial therapy in differentiated thyroid cancer: preablation-stimulated thyroglobulin better than the TNM-stage. Nucl Med Commun 2018; 39: 405-410.

48. Zheng CM, Ji YB, Song CM et al. Number of metastatic lymph nodes and ratio of metastatic lymph nodes to total number of retrieved lymph nodes are risk factors for recurrence in patients with clinically node negative papillary thyroid carcinoma. Clin Exp Otorhinolaryngol 2018; 11: 58-64. 